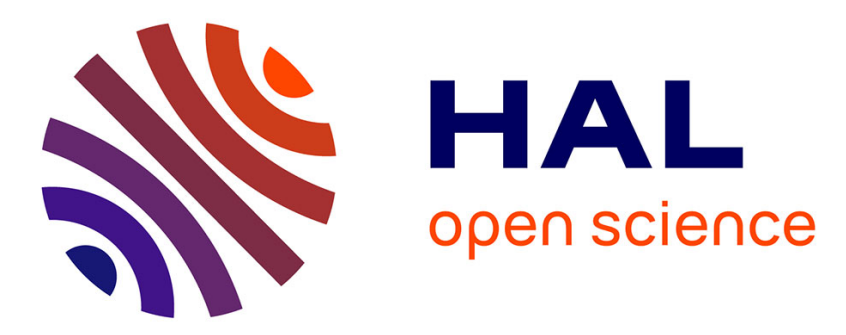

\title{
Étude par des méthodes calorimétriques d'une décharge haute fréquence dans de l'argon à la pression atmosphérique
}

\author{
Jean Besombes-Vailhé
}

\section{- To cite this version:}

Jean Besombes-Vailhé. Étude par des méthodes calorimétriques d'une décharge haute fréquence dans de l'argon à la pression atmosphérique. Revue de Physique Appliquée, 1967, 2 (4), pp.225-234. 10.1051/rphysap:0196700204022500 . jpa-00242796

\section{HAL Id: jpa-00242796 https://hal.science/jpa-00242796}

Submitted on 1 Jan 1967

HAL is a multi-disciplinary open access archive for the deposit and dissemination of scientific research documents, whether they are published or not. The documents may come from teaching and research institutions in France or abroad, or from public or private research centers.
L'archive ouverte pluridisciplinaire HAL, est destinée au dépôt et à la diffusion de documents scientifiques de niveau recherche, publiés ou non, émanant des établissements d'enseignement et de recherche français ou étrangers, des laboratoires publics ou privés. 


\title{
REVUE DE PHYSIQUE APPLIQUÉE
}

\author{
Supplément au " Journal de Physique "
}

\section{ÉTUDE PAR DES MÉTHODES GALORIMÉTRIQUES D'UNE DÉGHARGE HAUTE FRÉQUENGE DANS DE L'ARGON A LA PRESSION ATMOSPHÉRIQUE}

\author{
Par JEAN BESOMBES-VAILHÉ (1), \\ Centre de Spectrographie : Nouvelle Faculté des Sciences, I18, route de Narbonne, Toulouse, \\ et Institut du Génie Chimique : Chemin de la Loge, Toulouse.
}

\begin{abstract}
Résumé. - Nous avons étudié les puissances mises en jeu dans le jet d'une torche à plasma d'argon haute fréquence $(6,7 \mathrm{MHz})$ à la pression atmosphérique. Nous avons mesuré, par des méthodes calorimétriques, la puissance que l'on peut récupérer dans l'inducteur sous forme de chaleur, la puissance thermique dégagée par la décharge et, enfin, la puissance rayonnée dans l'intervalle spectral $4500 \AA$ à $6500 \AA$.

Nous avons calculé la température moyenne calorimétrique des gaz de la décharge et nous avons constaté que sa valeur est très inférieure $(30 \%)$ à celle du plasma déterminée par des méthodes spectrales. Nous avons vérifié à partir de la variation de cette température en fonction de la puissance rayonnée que, dans l'intervalle spectral envisagé, le rayonnement continu du plasma peut s'interpréter d'une façon satisfaisante $(10 \%)$ à partir de la théorie de W. Finkelnburg et Th. Peters [1]. Dans ces conditions et par application de l'équation de Saha [2], il en résulte que la puissance continue rayonnée par le plasma d'argon par atome n'est fonction que de sa température.
\end{abstract}

Abstract. - The power developed in the jet of a high frequency plasma torch $(6.7 \mathrm{MHz})$ at atmospheric pressure has been studied. The recoverable power in the inductor in the form of heat, the thermal power evolved by the discharge and, lastly, the radiated power in the spectral range of $4500 \AA$ to $6500 \AA$ have been measured by calorimetric methods.

The average calorimetric temperature of the discharge gases is calculated and it is shown that its value is much below $(30 \%)$ that of the plasma determined by spectral methods. From variation of temperature versus the radiated power we have checked that, in the spectral range considered, continuous plasma radiation may be explained satisfactorily $(10 \%)$ from W. Finkelnburg's and Th. Peters theory [1]. In this case and by applying the Saha equation [2], its follows that the continuous radiated power by an argon plasma atom is a function of its temperature only.

I. Introduction. - Pour mesurer les puissances mises en jeu au cours d'une décharge électrique, de nombreux auteurs ([3] à [14]) ont déjà eu recours aux méthodes calorimétriques en raison de leur sûreté et de leur commodité d'emploi. Nous citerons plus particulièrement, dans le cas de décharge inductive haute fréquence à la pression atmosphérique ([15] à [19]), les travaux de S. V. Dresvin, A. V. Donskoi et V. M. Gol'Dfarb [20] ainsi que ceux de B. M. Dimchitz et J. P. Koretski [21]. Ces auteurs ont mesuré la puissance dissipée dans des torches à plasma haute fréquence présentant des conditions de fonctionnement différentes de la nôtre : fréquence quatre fois plus

(1) Cet article recouvre en partie la Thèse de Doctorat d'Etat ès Sciences Physiques de l'auteur, Toulouse, 1968. grande, découplage important de l'inducteur par rapport au plasma, mode de refroidissement et caractéristiques électriques différents.

Le montage expérimental que nous avons employé est constitué par une décharge inductive haute fréquence $(6,7 \mathrm{MHz})$ à la pression atmosphérique dans une enceinte tubulaire (diamètre $24-27 \mathrm{~mm}$ ) en silice vitreuse et avec de l'argon comme fluide plasmagène. Nous avons examiné, en premier lieu, les puissances qui entrent en jeu au cours de la décharge et nous les avons mesurées par des méthodes calorimétriques. Nous avons étudié ensuite leurs variations en fonction de la tension plaque continue d'alimentation et du débit d'argon. Nous avons déterminé enfin une température moyenne des gaz de la décharge et caractérisé la variation de la puissance rayonnée par le plasma en 
fonction de la température mesurée par des méthodes spectrales. Les domaines de variation de nos essais ont été limités, d'une part, par l'extinction de la flamme et, d'autre part, par la destruction du tube à décharge, alors que nous n'avions pas atteint la tension continue plaque maximale du générateur.

Le fonctionnément du générateur haute fréquence est tel que les variations de la fréquence d'oscillation et de l'intensité grille en charge, c'est-à-dire lorsque la décharge est amorcée, sont faibles (respectivement 6 et $10 \%$ au maximum dans le domaine de nos essais). Aussi, de manière à éviter leurs influences, nous les avons maintenues constantes de sorte que nous n'avons disposé, pour effectuer nos essais, que de deux variables : la tension continue plaque d'alimentation et le débit d'argon.

II. Etude théorique. - 1. ÉtUde DES PUISSANGes MiSES EN JEU AU GOURS DE LA DÉGHARGE. - Dans un article précédent [22], nous avons décrit cette décharge et ses conditions de fonctionnement. Aux bornes de l'inducteur, le générateur nous fournit une puissance haute fréquence qui, transmise en partie au plasma, va servir à élever la température des gaz, à provoquer leur dissociation en atomes, à augmenter l'énergie interne des atomes et même à les ioniser. Remarquons que, dans le cas de la décharge dans de l'argon, gaz monoatomique, n'interviennent pas les phénomènes de dissociation, ce qui se traduit par une faible capacité calorifique des gaz de la décharge. La puissance haute fréquence étant difficile à mesurer par les méthodes électriques classiques, nous avons préféré caractériser énergétiquement le générateur par la puissance $P_{\mathrm{I}}$ à l'inducteur qui correspond à la puissance que l'on peut y récupérer sous forme de chaleur au moyen d'une charge métallique cylindrique placée entre les spires de l'inducteur et dans les mêmes conditions de fonctionnement électrique. Cette charge métallique reproduit exactement la charge du plasma [23], de sorte que $P_{\mathrm{I}}$ correspond à la puissance totale $P_{\mathrm{T}}$ que peuvent fournir les gaz de la décharge au milieu extérieur sous forme de conduction de convection ou de rayonnement.

Pour caractériser les conditions de fonctionnement électrique à intensité grille en charge constante du générateur, nous avons défini une puissance électrique $P_{\mathrm{E}}$ qui correspond à la différence des puissances électriques continues consommées par l'oscillateur, la décharge étant et n'étant pas amorcée. L'inducteur étant sous tension et la décharge n'étant pas amorcée, l'oscillateur à vide consomme une certaine puissance électrique à vide $P_{\mathrm{v}}$ que nous caractérisons par le produit de la tension plaque continue à vide par l'intensité plaque à vide. Nous faisons de même dans le cas de l'oscillateur en charge et définissons ainsi une puissance électrique en charge $P_{\mathrm{C}}$. La puissance électrique $P_{\mathrm{E}}$ sera donnée par la relation :

$$
\begin{gathered}
P_{\mathrm{E}}=P_{\mathrm{C}}-P_{\mathrm{V}} \\
P_{\mathrm{E}}=V_{2} \cdot I_{\mathrm{p}_{2}}-V_{1} \cdot I_{\mathrm{p}_{1}} .
\end{gathered}
$$

Nous avons étudié, dans un intervalle spectral déterminé (4500 $\AA<\lambda<6500 \AA$ ), une partie seulement de la puissance totale $P_{\mathrm{RT}}$ rayonnée par la flamme que nous avons appelée puissance rayonnée $P_{\mathrm{R}}$. Nous l'avons mesurée au moyen d'une lunette à radiation totale de la Société M.E.C.I. Cette lunette comporte, entre autres, une fenêtre de protection en silice vitreuse transparente devant laquelle nous avons placé un filtre vert nous définissant le domaine spectral utilisé.

Le tableau suivant schématise les transformations des diverses puissances envisagées au cours de la décharge : Puissance électrique $P_{\mathrm{E}} \rightarrow \begin{aligned} & \text { Puissance } P_{\mathrm{I}} \\ & \text { à l'inducteur }\end{aligned} \rightarrow \begin{aligned} & \text { Puissance } \\ & \text { totale } P_{\mathrm{T}}\end{aligned}$

En désignant par $P_{\mathrm{G}}$ la puissance restant aux gaz de la décharge, nous avons :

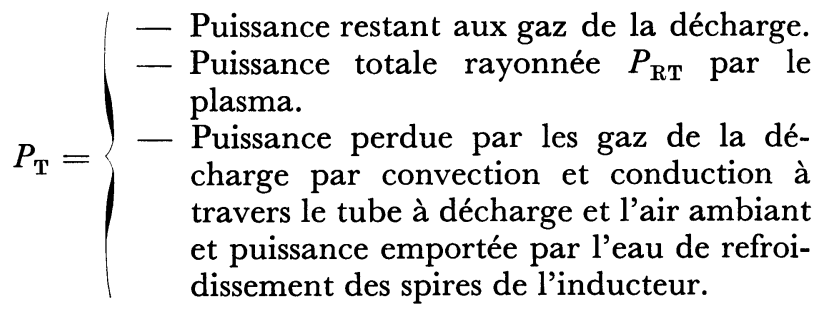

2. DÉtermination DE LA TEMPÉRATURe MOYenNe DES GAZ DE LA DÉGHARGE A PARTIR DE MESURES GALORIMÉtriQUES. - La puissance totale $P_{\mathrm{T}}$ que nous avons définie ne correspond pas exactement à la puissance $P_{\mathrm{K}}$ que nous avons mesurée avec le calorimètre métallique englobant toute la décharge, car nous n'avons pas fait intervenir dans nos mesures la puissance calorifique emportée par l'eau de refroidissement de l'inducteur. Cette puissance calorifique se décompose en deux, l'une $P_{\mathrm{J}}$, provenant de la chaleur produite dans l'inducteur par effet Joule, et l'autre $P_{\mathrm{D}}$, provenant du refroidissement du tube à décharge. La première puissance n'intervient pas dans l'évaluation de $P_{\mathrm{T}}$, alors que la seconde $\mathrm{y}$ joue son rôle et y réprésente une valeur non négligeable de l'ordre de $12 \%$ de $P_{\mathrm{T}}$. Remarquons, en outre, que nos mesures calorimétriques n'ont pas fait intervenir la chaleur transmise par conduction dans le sens longitudinal à l'intérieur du tube en silice vitreuse. Cette perte de puissance est très faible, de l'ordre d'une dizaine de watts, étant donné le faible coefficient de conductibilité thermique de la silice vitreuse [24]. De plus, nous n'avons pas tenu compte de la chaleur rayonnée vers l'extérieur par le trou d'introduction du tube à décharge. Ce trou correspond, par rapport au centre de la flamme, à un angle solide $\Omega=2 \pi(1-\cos \theta)$, soit 0,036 stéradian, ce qui entraîne une perte de puissance égale à $0,7 \%$ de la puissance rayonnée. Ces deux dernières pertes sont négligeables par rapport à la puissance mesurée.

Pour calculer la température moyenne calorimétrique des gaz, nous avons effectué leur bilan énergétique entre l'entrée et la sortie du tube à décharge en supposant que le plasma y a pratiquement reçu toute l'énergie électromagnétique de l'inducteur. Pour pou- 
voir appliquer cette hypothèse, nous avons augmenté la longueur du tube à décharge après les spires de l'inducteur $(5 \mathrm{~cm})$ de manière à y contenir tout le plasma. Nous avons pu calculer ainsi l'enthalpie moyenne des gaz à la sortie du tube et déterminer ensuite leur température moyenne. Le bilan exprimé par rapport aux puissances s'écrit :

Puissance totale $P_{\mathrm{T}}\left\{\begin{array}{l}\text { - Puissance calorimétrique } P_{\mathrm{K}} \\ \text { - Puissance } P_{\mathrm{D}} \text { emportée par } \\ \text { l'eau de refroidissement de } \\ \text { l'inducteur. }\end{array}\right.$ - Puissance $P_{\mathrm{G}}$ restant aux gaz de la décharge à la sortie du tube.

- Puissance totale $P_{\mathrm{RT}}$ rayonnée par le plasma.

- Puissance perdue $P_{\mathrm{p}}$ par les gaz de la décharge par conduction et convection à travers le tube à décharge.

soit : $P_{\mathrm{G}}=P_{\mathrm{K}}-P_{\mathrm{RT}}-P_{\mathrm{P}}$.

Nous avons mesuré $P_{\mathrm{RT}}$ en appliquant la méthode du calorimètre opaque et transparent décrite par S. V. Dresvin et ses collaborateurs [20]. Nous avons négligé, en première approximation, l'absorption du rayonnement par la silice vitreuse et l'eau de refroidissement.

La puissance $P_{\mathrm{P}}$ perdue par les gaz de la décharge à travers le tube équivaut approximativement à la perte de chaleur par unité de temps entre un tube de $27 \mathrm{~mm}$ de diamètre extérieur, de $20 \mathrm{~cm}$ de long et de température extérieure moyenne de paroi tp égale à $470^{\circ} \mathrm{C}$, plongé dans de l'air calme à la température moyenne $t_{\mathrm{a}}$ de $20^{\circ} \mathrm{C}$. Nous avons mesuré la température de la paroi extérieure du tube au moyen d'une sonde thermométrique équipée d'un thermocouple $\mathrm{Pt}-\mathrm{Pt} \mathrm{Rd}$ et nous avons obtenu une valeur moyenne de tp en effectuant la moyenne logarithmique des valeurs mesurées. Dans ces conditions, $P_{\mathrm{P}}$ peut s'écrire :

$$
P_{\mathrm{P}}=\left(h_{\mathrm{o}}+h_{\mathrm{R}}\right) \cdot A_{\mathrm{S}} \cdot\left(t_{\mathrm{p}}-t_{\mathrm{a}}\right) \cdot 4,18 / 3600
$$

$h_{\mathrm{o}}$ : coefficient d'échange par convection naturelle en $\mathrm{kcal} / \mathrm{h} \cdot \mathrm{m}^{2} \cdot{ }^{\circ} \mathrm{C}$.

$h_{\mathrm{R}}$ : coefficient de transmission de chaleur par rayonnement en $\mathrm{kcal} / \mathrm{h} \cdot \mathrm{m}^{2} \cdot{ }^{\circ} \mathrm{C}$.

$A_{\mathrm{S}}$ : surface d'échange extérieure en $\mathrm{m}^{2}$.

Nous avons employé pour calculer le coefficient $h_{0}$ la relation établie par W. H. McAdams [25], soit :

$$
h_{\mathrm{o}}=1,13(\Delta t / D)^{0,25}
$$

$\Delta t$ : différence de température en ${ }^{\circ} \mathrm{C}$ entre la surface chaude du tube et l'air froid.

$D$ : diamètre extérieur du tube en $\mathrm{m}$.

L'expression donnant $h_{\mathrm{R}}$ est de la forme :

$$
h_{\mathrm{R}}=\frac{4,92 \cdot \varepsilon_{\mathrm{S}} \cdot\left[\left(T_{\mathrm{p}} / 100\right)^{4}-\left(T_{\mathrm{a}} / 100\right)^{4}\right]}{\left(T_{\mathrm{p}}-T_{\mathrm{a}}\right)}
$$

$\varepsilon_{\mathrm{S}}$ : facteur d'émission normal global de la surface. Nous l'avons pris égal à 0,95 [26].

$T_{\mathrm{p}}$ : température absolue en ${ }^{\circ} \mathrm{K}$ de la paroi du tube à décharge.

$T_{\mathrm{a}}$ : température absolue de l'air ambiant en ${ }^{\circ} \mathrm{K}$.
Si $M$ désigne le débit d'argon en kmole/s, l'enthalpie molaire $H$ de l'argon à la sortie du tube en $\mathrm{kcal} / \mathrm{kmole}$ s'écrit :

$$
H=\frac{P_{\mathrm{G}}}{4,18 M}+H_{0}
$$

$H_{0}$ : enthalpie molaire de l'argon à l'entrée du tube à décharge, c'est-à-dire à $20^{\circ} \mathrm{C}$, soit : $H_{0}=0,143 \times 10^{4} \mathrm{kcal} / \mathrm{kmole}$ [27], en prenant l'origine des enthalpies à 0 oK.

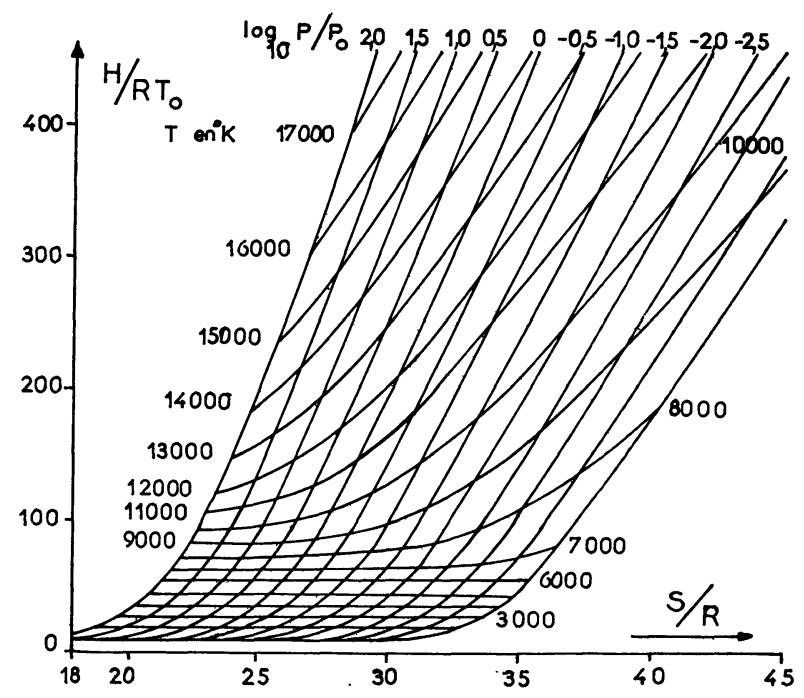

FIG. 1. - Variation pour l'argon des groupes adimensionnels $H / R T_{0}$ et $S / R$ pour différentes valeurs de la température et de la pression $P$.

( $H$ et $S$ : enthalpie et entropie de l'argon; $R$ : constante des gaz parfaits ; $T_{0}=273 \circ \mathrm{K} ; P_{0}=1 \mathrm{~atm}$. ; Origine des enthalpies à zéro degré Kelvin [27].)

Cette valeur nous a permis de calculer la température moyenne calorimétrique des gaz grâce aux isothermes du diagramme de la figure 1 [28].

3. ÉTUDE DE LA VARIATION DE LA PUISSANCE RAYONNÉE EN FONCTION DE LA TEMPÉRATURE. - Au cours de la décharge à la pression atmosphérique, nous avons 
constaté que les variations de la tension continue de la plaque ne modifiaient pratiquement pas les dimensions volumétriques de la flamme, alors que les variations du débit d'argon entraînaient, dans le même sens, une modification de ces dimensions. Nous avons donc supposé, pour en tenir compte, que les dimensions volumétriques de la flamme sont proportionnelles au débit $Q$ d'argon à la puissance $\alpha$. Ce résultat met en valeur l'hétérogénéité importante de la décharge par rapport aux atomes d'argon. Il faut, en effet, considérer d'une part ceux qui constituent le plasma et, d'autre part, ceux qui servent uniquement à refroidir le tube à décharge.

La puissance totale rayonnée par la source correspond en fait à la superposition de deux formes de rayonnement : d'une part, le rayonnement produit par les atomes d'argon et qui est caractérisé par les raies spectrales et, d'autre part, le rayonnement continu. Or, étant donné l'importance de l'intensité du spectre continu par rapport aux raies d'émission, nous avons fait l'hypothèse, dans le domaine spectral utilisé où la plupart des raies intenses de l'argon sont éliminées, que la puissance rayonnée par le spectre de raies est faible par rapport à la puissance continue $P_{\mathrm{RC}}$ rayonnée par le plasma, c'est-à-dire que nous avons supposé $P_{\mathrm{R}}$ peu différent de $P_{\mathrm{RC}}$. Les travaux de V. M. Gol'Dfarb ([29] et [30]), de E. S. Borovick et de ses collaborateurs ([31] et [32]) confirment cette hypothèse. Ces auteurs indiquent que, pour des températures voisines de $10000^{\circ} \mathrm{K}$ et pour des pressions supérieures ou égales à la pression atmosphérique, la contribution du rayonnement du spectre de raies est faible par rapport à celle du rayonnement total.

Comme H. Triche [33] a caractérisé le rayonnement produit par les atomes par la puissance disponible par atome, nous avons caractérisé le rayonnement continu du plasma par la puissance continue $P_{\mathrm{RV}}$ rayonnée par unité de volume dans les conditions de la décharge. Il existe trois mécanismes de rayonnement d'un plasma : le rayonnement dû aux collisions électronatome neutre, électron-électron et électron-ion. Le premier mécanisme n'intervient pas dans la décharge, car les fréquences de collisions électron-atome neutre sont négligeables ([34] et [35]). Le deuxième mécanisme ne représente un effet sensible que pour des températures très élevées $(T>107 \mathrm{o})$. Quant au troisième mécanisme, il se décompose en deux [36]. Le premier correspond à un rayonnement de bremsstrahlung et le second à un rayonnement de recombinaison radiative. F. Gabannes et ses collaborateurs ([34] et [35]) ont indiqué, dans le cas d'un plasma d'argon ayant une température analogue à celle que nous avons obtenue $(10000 \mathrm{~K})$, que, dans le visible, le rayonnement de recombinaison est le plus important, le bremsstrahlung ne correspondant qu'à une faible partie du rayonnement continu émis (20\%). La théorie de W. Finkelnburg et Th. Peters [1] leur a permis d'interpréter correctement leurs résultats. Les travaux de E. S. Borovick et de ses collabora- teurs ([30] et [31]) indiquent de même, dans ces domaines de températures, que le mécanisme le plus important du rayonnement est la recombinaison. Or, étant donné que la fréquence limite $\delta g$ pour l'argon $\left(\delta g=0,65 \times 10^{5} \mathrm{~Hz}\right.$, soit $\left.\lambda g=4610 \AA\right)$ [37] se trouve juste à la limite supérieure du domaine de transparence du filtre d'entrée de la lunette, nous avons supposé, en première approximation, que nous avons analysé un rayonnement dont la longueur d'onde $\lambda$ est supérieure à $\lambda g$. Dans ces conditions, la puissance $P_{\mathrm{RV}}$ rayonnée par l'unité de volume est donnée [35] en fonction de la densité électronique $n_{\mathrm{e}}$ et de la température électronique $T_{\mathrm{e}}$ par la relation :

$$
P_{\mathrm{RV}}=K_{0} \cdot n_{\mathrm{e}}^{2} \cdot T_{\mathrm{e}}^{-1 / 2}
$$

où $K_{0}$ est une constante caractéristique du fluide plasmagène.

Nous n'avons pas fait intervenir dans l'expression précédente (2) le facteur correctif $g(\lambda)$ de Gaunt [38], car, dans l'intervalle spectral envisagé, sa valeur est voisine de 1 , alors qu'elle peut atteindre des valeurs élevées dans l'infrarouge lointain [39]. L'expression (2) est analogue à celle utilisée par V. M. Gol'Dfarb ([29] et [30]) dans le cas d'un plasma d'argon, et qu'il a obtenue après avoir effectué les corrections de L. M. Biberman et G. E. Norman [40] dans le cadre du modèle proposé par Kramer ([41] et [42]).

Dans l'intervalle spectral utilisé (4500 $\AA<\lambda<$ $6500 \AA)$, nous avons donc pu écrire la puissance continue rayonnée sous la forme :

$$
P_{\mathrm{R}}=K_{1} \cdot R_{\mathrm{RV}} \cdot Q^{\alpha}
$$

où $K_{1}$ est une constante caractéristique des dimensions volumétriques du plasma.

Nous avons fait, en outre, l'hypothèse de l'équilibre thermodynamique local, de sorte que nous avons pris $T_{\mathrm{e}}$ égal à $T_{\mathrm{s}}$, température moyenne du plasma déterminée par les méthodes spectrales. Nous avons utilisé l'élargissement de la raie $H_{\beta}$ de l'hydrogène [22].

L'expression (2) s'écrit :

$$
\log _{10} P_{\mathrm{R}}=K+\log _{10}\left(n_{\mathrm{e}}^{2} \cdot T_{\mathrm{s}}^{-1 / 2}\right)+\alpha \cdot \log _{10} Q
$$

où $K$ est une constante caractéristique du fluide.

Nous avons employé les résultats de A. Sy [43], de K. S. Drellishak et de ses collaborateurs [44] pour obtenir $n_{\mathrm{e}}$ en fonction de la température.

III. Etude expérimentale. - Nous allons examiner séparément pour chaque mesure de puissances envisagées son dispositif expérimental et les résultats obtenus.

1. Mesure de la puissance a l'inducteur $P_{\mathrm{I}}$. Pour effectuer cette mesure, nous avons placé une charge métallique entre les spires de l'inducteur sui- 


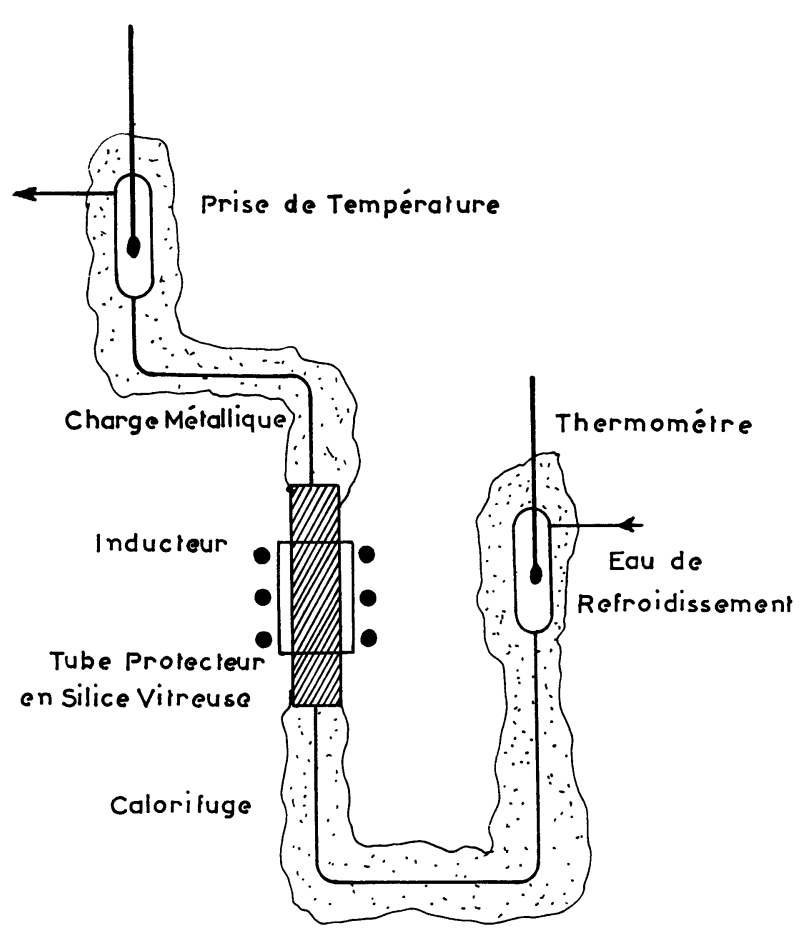

FIG. 2. - Schéma descriptif

de la mesure de la puissance $P_{I}$ à l'inducteur.

vant le schéma tracé sur la figure 2 . Ces charges sont constituées d'un tube cylindrique en acier ordinaire de $80 \mathrm{~mm}$ de long, d'épaisseur supérieure ou égale au minimum à $1 \mathrm{~mm}$ et de diamètre extérieur variable compris entre 4 et $18 \mathrm{~mm}$. Elles sont protégées des spires de l'inducteur grâce à un tube en silice vitreuse (diamètre 24-27 $\mathrm{mm}$ ) de $6 \mathrm{~cm}$ de long. La mesure du débit de l'eau de refroidissement est réalisée au moyen d'un manomètre différentiel à eau relié à un diaphragme répondant aux normes de l'A.F.N.O.R. Nous avons choisi pour mesurer la température d'entrée et de sortie de l'eau des thermomètres à mercure au 1/100 de degré que nous avons placés suffisamment loin de l'inducteur ( $30 \mathrm{~cm}$ environ) pour que l'action du champ haute fréquence soit négligeable.

La chaleur produite dans les charges est véhiculée par l'eau de refroidissement dont on règle le débit de façon que le gradient de température soit faible $\left(\Delta t \leqslant 3^{\circ} \mathrm{G}\right)$. Nous avons calorifugé sommairement le montage car, d'une part, nous avons effectué nos mesures avec un gradient de température faible entre l'eau de refroidissement et l'air ambiant $\left(\Delta t \leqslant 5{ }^{\circ} \mathrm{G}\right)$ et, d'autre part, notre ensemble calorimétrique présente une faible surface d'échange avec l'extérieur $\left(s=0,05 \mathrm{~m}^{2}\right)$. Comme nous mesurons des différences de température et que ces différences sont faibles, les corrections thermométriques sont peu élevées.

De la mesure des températures d'entrée et de sortie de l'eau de refroidissement, de son débit et des caractéristiques électriques, nous avons calculé $P_{\mathrm{I}}$ et $P_{\mathrm{E}}$.
Nous avons porté sur le graphique ( fig. 3) leur variation en caractérisant chaque charge par un signe distinctif déterminé. Le résultat obtenu montre que les différents points de mesure correspondant à chacune

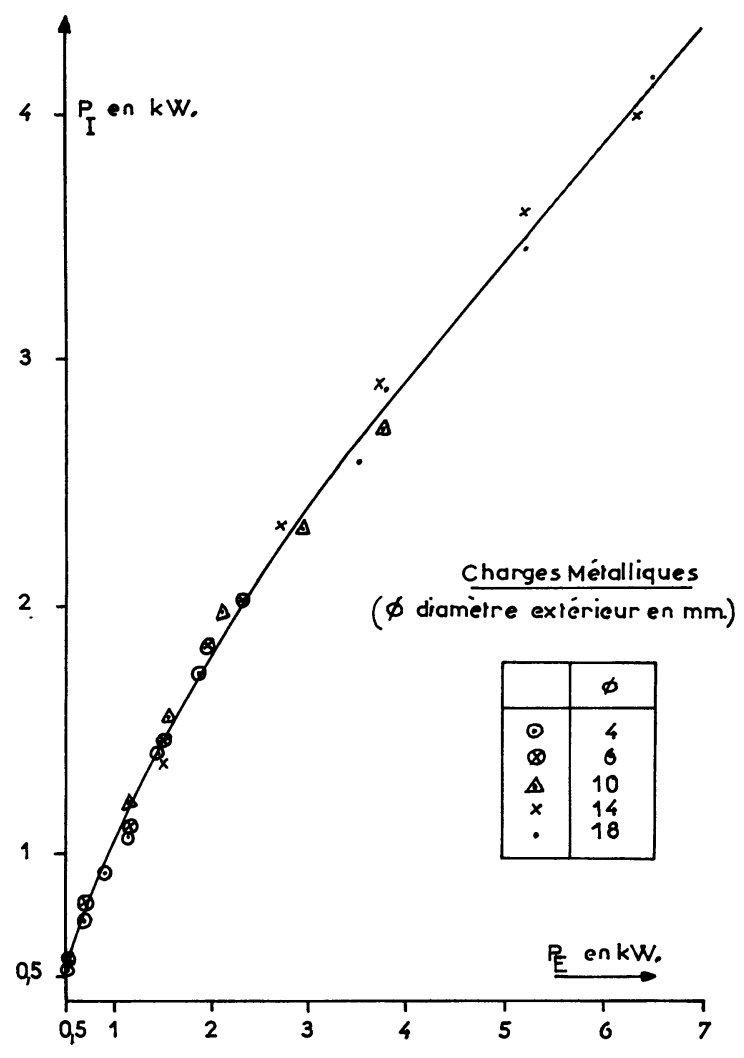

FIG. 3. - Variation de la puissance $P_{I}$ à l'inducteur en fonction de la puissance électrique $P_{\mathbf{E}}$. (A chaque charge métallique correspond un signe distinctif déterminé.)

des charges se raccordent assez bien pour former une courbe unique. Nous avons conclu que la puissance électrique $P_{\mathrm{E}}$ précédemment définie caractérise énergétiquement le plasma et nous l'avons employée comme puissance de référence, car sa mesure est commode et facile.

2. Mesure de la puissance GalorimétriQue $P_{\mathrm{K}}$. Gette puissance est recueillie à l'aide d'un calorimètre à circulation d'eau placé autour de la décharge suivant le schéma de la figure 4. Cet appareil est formé essentiellement de trois parties :

- une partie supérieure constituée par une chambre métallique circulaire à double paroi;

- une partie inférieure constituée par un échangeur à faisceau tubulaire de 30 tubes de cuivre (diamètres $4-6 \mathrm{~mm}$ );

- une dernière partie formée d'un calorifuge en laine de verre entourant tout l'ensemble. 


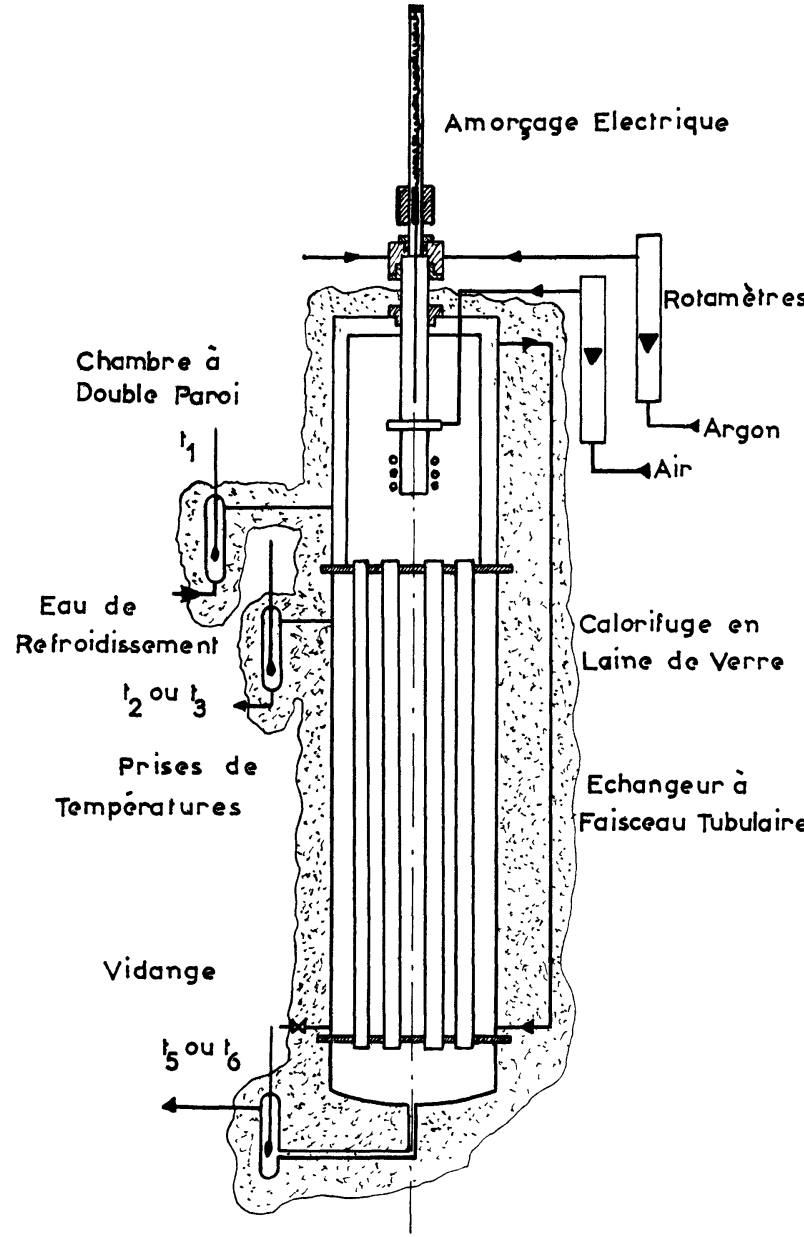

FIG. 4. - Schéma descriptif du calorimètre.

L'appareil ainsi conçu ne fonctionne que l'espace d'une seconde car, dès la mise en tension, l'inducteur est le siège de décharges électriques dues à la présence d'argon autour de ses spires. Nous avons éliminé ce dernier en soufflant de l'air autour de l'inducteur. Dans ces conditions, l'appareil fonctionne parfaitement. Les débits d'air $\left(2 \mathrm{~m}^{3} / \mathrm{h}\right.$ environ) et d'argon introduits dans la chambre sont mesurés par des rotamètres. Le débit d'eau est réglé de telle façon que son élévation de température soit faible $\left(\Delta t \leqslant 3^{\circ} \mathrm{C}\right)$. L'efficacité du calorifuge est telle que les pertes de chaleur sont négligeables.

A partir de la mesure des températures d'entrée et de sortie de l'eau et des gaz (argon et air) introduits dans le calorimètre, la décharge étant et n'étant pas amorcée, ainsi que des débits volumiques correspondants, nous avons déterminé la puissance calorimétrique $P_{\mathrm{K}}$ en faisant varier le débit d'argon et la différence de potentiel plaque continue. Nous avons tracé sur le graphique (fig. 5) la variation de $P_{\mathrm{K}}$ en fonction de $P_{\mathrm{E}}$ obtenu après correction de la puissance perdue par induction dans le corps du calorimètre $\left(4,5 \%\right.$ de $P_{\mathrm{E}}$ au maximum). Ce graphique montre que, d'une part, $P_{\mathrm{K}}$ et $P_{\mathrm{E}}$ sont, en première approximation, indépendants du débit d'argon et, d'autre part, pour des valeurs de $P_{\mathrm{K}}$ comprises entre 2 et $4,2 \mathrm{~kW}$, que la courbe représentative est approximativement une droite. Ce résultat peut se traduire par la forme algébrique suivante :

$$
P_{\mathrm{K}}=0,46 \cdot P_{\mathrm{E}}+0,74
$$

$P_{\mathrm{K}}$ et $P_{\mathrm{E}}$ en kilowatts avec $2 \mathrm{~kW}<P_{\mathrm{K}}<4,2 \mathrm{~kW}$.

Le graphique précédent représente une moyenne des mesures effectuées. On peut cependant remarquer que $P_{\mathrm{K}}$ a légèrement tendance à augmenter avec le débit d'argon, alors que $P_{\mathrm{E}}$ a tendance à diminuer. Remarquons, en outre, que la différence entre $P_{\mathrm{I}}$ et $P_{\mathrm{K}}$

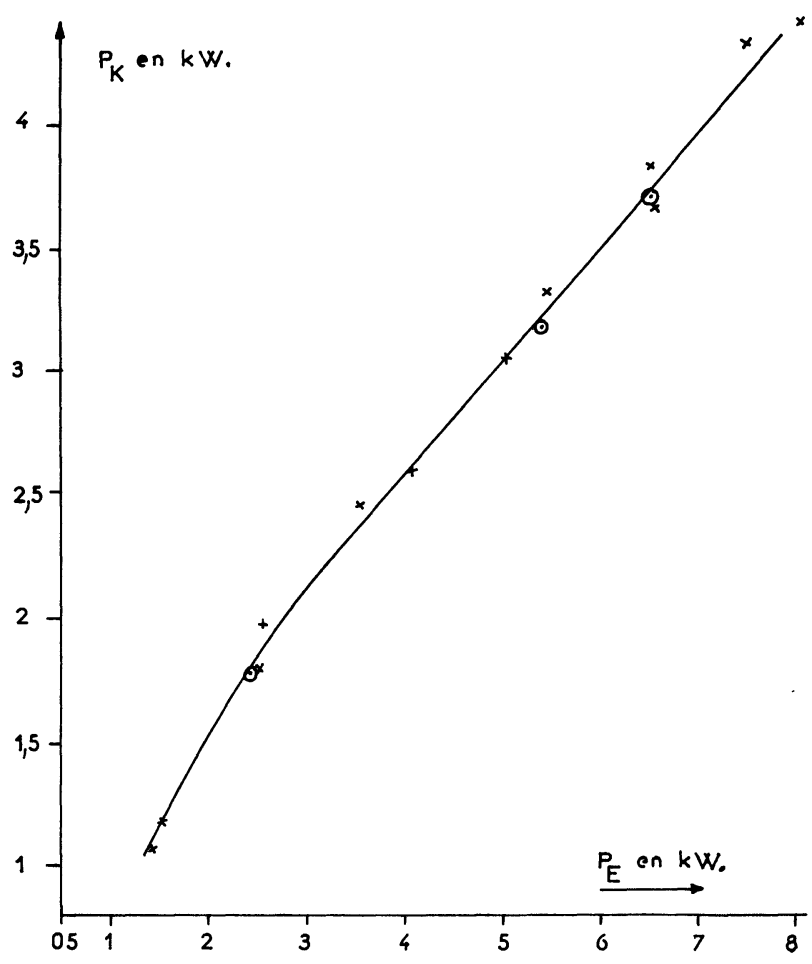

FIG. 5. - Variation de la puissance calorimétrique $P_{\mathrm{K}}$ en fonction de la puissance électrique $P_{\mathrm{E}}$. (Les points du graphique représentés par $\odot$ correspondent à une moyenne de 6 essais.)

pour une valeur donnée de $P_{\mathrm{E}}$ correspond à la puissance $P_{\mathrm{D}}$ emportée par l'eau de refroidissement de l'inducteur.

3. Mesure de la puissance rayonnée $P_{\mathrm{R}}$. - Pour effectuer cette mesure, nous avons utilisé une lunette à radiation totale de la Société M.E.C.I., $\mathrm{n}^{0} \mathrm{RTM}$, 2163. Cette lunette est formée essentiellement de deux miroirs sphériques protégés par une fenêtre en silice fondue transparente devant laquelle est placé le filtre vert. Ces miroirs concentrent l'énergie rayonnée sur une thermophile constituée de huit soudures chaudes de thermocouple $(\mathrm{Pt}-\mathrm{Pt} . \mathrm{Rd})$ créant une force élec- 
tromotrice approximativement proportionnelle à la puissance reçue par la thermopile [45], donc à la puissance rayonnée.

Nous avons placé la lunette verticalement dans l'axe de la flamme et à une distance de visée de $60 \mathrm{~cm}$ (position conseillée par le constructeur). Dans ces conditions, tout le rayonnement émis par la flamme vers le haut dans le sens vertical pénètre dans la lunette, de sorte que la mesure tient compte des variations éventuelles des dimensions volumiques du plasma. La force électromotrice obtenue est mesurée au moyen d'un enregistreur Speedomax M.E.C.I. dont les circuits ont été protégés de l'action du champ haute fréquence. Pour éliminer l'influence du filtre sur l'intensité du rayonnement, nous avons multiplié la force électromotrice mesurée par l'inverse du coefficient moyen de transmission du filtre ( 2 environ). Nous avons tracé sur le graphique ( $f g .6)$ en fonction de $P_{\mathrm{E}}$,

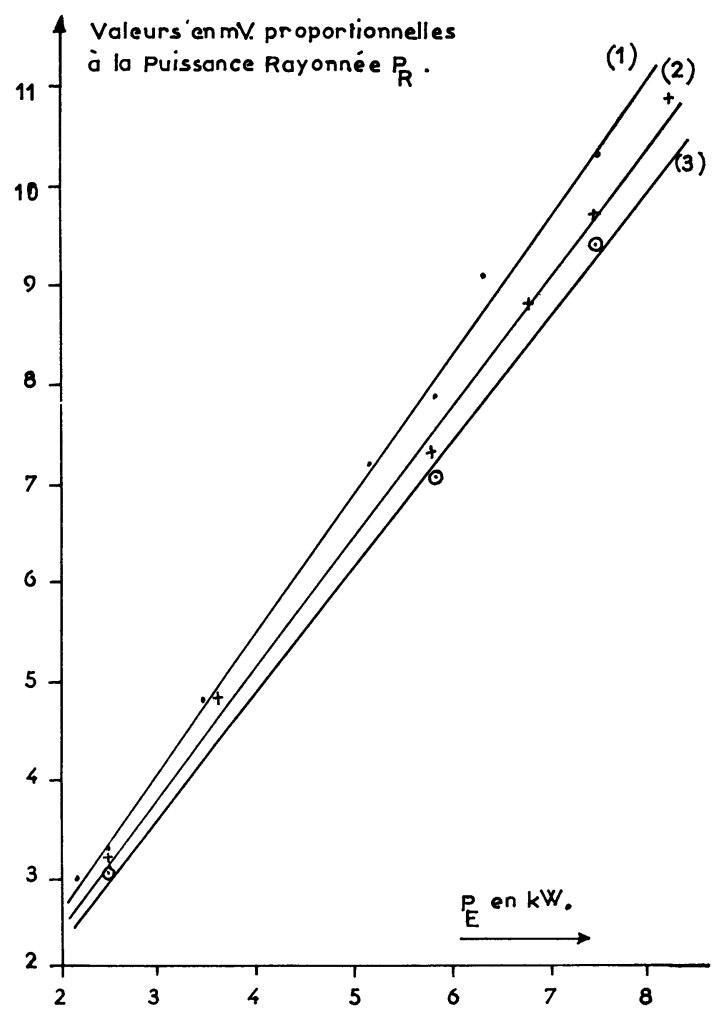

FIG. 6. - Variation de la puissance rayonnée $P_{R}$ en fonction de la puissance électrique $P_{\mathbf{E}}$ et du débit d'argon $Q$. (Les courbes 1,2 et 3 correspondent à des débits d'argon respectifs de 2,12, 1,67 et 1,42 $\mathrm{m}^{3} / \mathrm{h}$.)

et pour différentes valeurs du débit d'argon, la variation de cette force électromotrice approximativement proportionnelle à $P_{\mathrm{R}}$. Nous avons obtenu pratiquement, dans le domaine d'étude $\left(2 \mathrm{~kW}<P_{\mathrm{E}}<8 \mathrm{~kW}\right)$, un faisceau de droites où chacune d'elles correspond à une valeur déterminée du débit d'argon. Ce résultat nous montre que, dans le domaine spectral envisagé, d'une part la puissance rayonnée est une fonction linéaire de la puissance électrique et, d'autre part, qu'elle augmente avec le débit d'argon à puissance électrique constante.

Nous avons mesuré, en outre, en valeur absolue la puissance totale $P_{\mathrm{RT}}$ rayonnée en appliquant la méthode du calorimètre opaque et transparent [20]. Nous avons obtenu une variation linéaire en fonction de la puissance électrique qui est analogue à celle du graphique (fig. 6). Pour une valeur de $P_{\mathrm{E}}=5,5 \mathrm{~kW}$, nous avons obtenu $P_{\mathrm{RT}}=0,52 \mathrm{~kW}$, soit $14,3 \%$ de $P_{\mathrm{I}}$. Ge résultat est tout à fait en harmonie avec celui de M. P. Sherman et de ses collaborateurs ([47] et [48]) qui, dans le cas d'un arc soufflé et pour des températures de $11000 \mathrm{~K}$, ont trouvé pour $P_{\mathrm{RT}}$ une valeur égale à $12 \%$ de la puissance nette induite.

4. Détermination de la température moyenNe GALORIMÉTRIQUe DES GAZ DE LA DÉGHARGe. - Nous avons effectué ce calcul à partir d'un des cas les plus couramment rencontrés au cours de nos expériences et défini par les données suivantes :

- Débit d'argon

$$
Q=1,42 \mathrm{~m}^{3} / \mathrm{h}=1,76 \times 10^{-5} \mathrm{kmole} / \mathrm{s} .
$$

- Puissance électrique $P_{\mathrm{E}}=5,5 \mathrm{~kW}$.

- Puissance calorimétrique $P_{\mathrm{K}}=3,27 \mathrm{~kW}$.

- Puissance totale rayonnée $P_{\mathrm{RT}}=0,52 \mathrm{~kW}$.

- Température $T_{\mathrm{s}}$ déterminée par voie spectrale : $10000 \mathrm{~K}$.

- Diamètre extérieur du tube à décharge : $27 \mathrm{~mm}$.

- Longueur utile du tube à décharge : $20 \mathrm{~cm}$.

- Température moyenne de la paroi $t_{\mathrm{p}}=470^{\circ} \mathrm{C}$.

- Température moyenne de l'air ambiant $t_{\mathrm{a}}=20^{\circ} \mathrm{C}$.

Nous avons calculé en premier lieu $P_{\mathrm{G}}$, soit :

$$
\begin{aligned}
& P_{\mathrm{RT}}=0,52 \mathrm{~kW}, \\
& P_{\mathrm{P}}=0,39 \mathrm{~kW}, \\
& P_{\mathrm{G}}=2,36 \mathrm{~kW} .
\end{aligned}
$$

Remarquons que $P_{\mathrm{P}}$ correspond à $11,9 \%$ de la puissance calorimétrique. Ce résultat nous fait entrevoir les pertes considérables de la décharge torche dans l'air ambiant.

D'où la valeur de $H$ :

$$
H=3,35 \times 10^{4} \mathrm{kcal} / \mathrm{kmole} \text {. }
$$

Pour utiliser le diagramme de la figure 1 , nous avons calculé le groupe adimensionnel $H / R T_{0}$ (l'origine des enthalpies est prise au $0{ }^{\circ} \mathrm{K}$ et $R T_{0}=$ $542,99 \mathrm{kcal} / \mathrm{kmole})$ et le rapport $\log _{10} P / P_{0}$ qui est égal à 0 car $P$, pression de marche, est égale à une atmosphère, soit $P_{0}$. D'où :

$$
\begin{aligned}
& H / R T_{0}=61,7 \\
& \log _{10} P / P_{0}=0 .
\end{aligned}
$$

On obtient ainsi une valeur de la température égale à $7000 \mathrm{oK}$. Ces calculs nous montrent l'influence considérable du débit d'argon sur la valeur $T_{\mathrm{K}}$ de 
la température moyenne calorimétrique des gaz, alors qu'il est pratiquement sans influence sur la puissance calorimétrique et sur la température $T_{\mathrm{s}}$ du plasma. De plus, nous constatoṇs une différence importante de $30 \%$ entre $T_{\mathrm{s}}$ et $T_{\mathrm{K}}$. Ces résultats mettent en évidence l'hétérogénéité importante de la décharge par rapport aux atomes d'argon. La température spectrale $T_{\mathrm{s}}$ caractérise surtout le plasma, alors que la température moyenne $T_{\mathrm{K}}$ caractérise l'ensemble des atomes d'argon. Il est donc logique que cette dernière température soit inférieure à $T_{\mathrm{s}}$ et qu'elle diminue, à puissance constante, avec le débit d'argon. Cette discordance entre les résultats obtenus par les méthodes optiques et calorimétriques a déjà été soulignée par les travaux de S. V. Dresvin et de ses collaborateurs [20], relatifs à la mesure de sections efficaces.

5. ÉtUde DE LA PUISSANGe RAYONNÉE. - Les courbes du graphique ( fig. 6) montrent, en premier lieu, que la puissance rayonnée diminue à puissance électrique constante avec le débit d'argon. Cette variation est approximativement constante et égale à $11 \%$ pour une variation de $32 \%$ de ce dernier. De plus, à débit d'argon constant, ce graphique permet de calculer $P_{\mathrm{R}}$ en fonction de $P_{\mathrm{E}}$.

Nous avons porté sur le graphique (fig. 7) les résultats des mesures de $T_{\mathrm{S}}$ en fonction de $P_{\mathrm{E}}$ et la courbe obtenue s'incurve très nettement vers le bas. De plus, nous constatons que, d'une part, le débit d'argon est sans influence sensible sur $T_{\mathrm{s}}$ et, d'autre part, la faible variation de la température moyenne du plasma eu égard à celle de $P_{\mathrm{E}}$. De la variation de $n_{\mathrm{e}}$ en fonction de $T_{\mathrm{s}}$ [24] nous avons calculé $n_{\mathrm{e}}^{2} \cdot T_{\mathrm{s}}^{-1 / 2}$ en fonction de $T_{\mathrm{s}}$. A partir de ce résultat et des graphiques

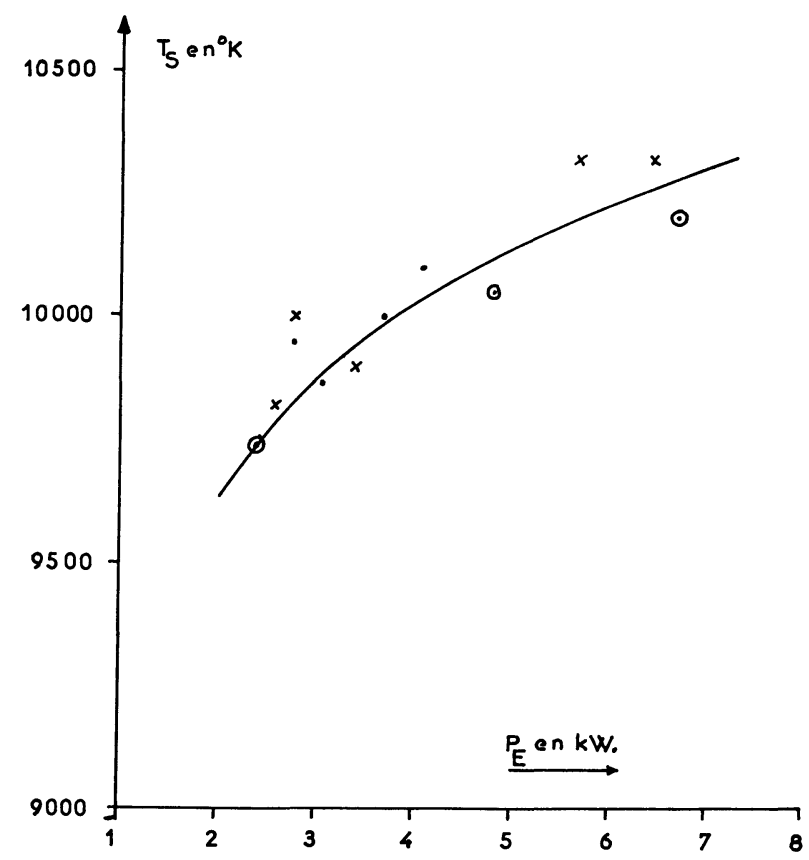

Frg. 7. - Variation de la température $T_{\mathrm{S}}$ du plasma en fonction de la puissance électrique $P_{\mathbf{E}}$. (Les points du graphique représentés par $\odot$ correspondent à une moyenne de 6 essais.)

(fig. 6 et 7), nous avons déterminé graphiquement, en éliminant $P_{\mathrm{E}}$ et $T_{\mathrm{S}}$, la variation de $\log _{10} P_{\mathrm{R}}$ à débit d'argon constant $\left(Q=1,42 \mathrm{~m}^{3} / \mathrm{h}\right)$ en fonction de $\log _{10}\left(n_{\mathrm{e}} T_{\mathrm{s}}^{-1 / 2} \times 10^{-30}\right)$. Les valeurs obtenues sont rapportées sur le tableau suivant :

\begin{tabular}{|c|c|c|c|c|}
\hline $\begin{array}{c}P_{\mathrm{E}} \\
\mathrm{EN} \quad \mathrm{kW}\end{array}$ & $\begin{array}{c}\text { FORGE } \\
\text { ÉLEGTROMOTRIGE } \\
\text { EN } \mathrm{mV}\end{array}$ & $\log _{10} P_{\mathrm{R}}$ & 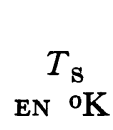 & $\log _{10}\left(n_{\mathrm{e}}^{2} \cdot T_{\mathrm{s}}^{-1 / 2} \cdot 10^{-30}\right)$ \\
\hline - & - & - & - & - \\
\hline 2,5 & 3,0 & 0,477 & 9760 & 0,172 \\
\hline 3,0 & 3,6 & 0,556 & 9870 & 0,256 \\
\hline 4,0 & 4,9 & 0,690 & 10020 & 0,374 \\
\hline 5,0 & 6,17 & 0,790 & 10130 & 0,460 \\
\hline 6,0 & 7,42 & 0,870 & 10220 & 0,536 \\
\hline 7,0 & 8,67 & 0,938 & 10300 & 0,595 \\
\hline
\end{tabular}

La courbe représentative est une droite de pente 1,1 . Nous avons effectué ce même calcul pour deux autres valeurs du débit d'argon $\left(1,67\right.$ et $\left.2,12 \mathrm{~m}^{3} / \mathrm{h}\right)$. Les pentes ainsi obtenues sont peu différentes de 1 à $10 \%$ près. Ce résultat assez satisfaisant vérifie $a$ posteriori les expressions (2) et (3) et, par là même, leurs hypothèses d'application. Nous pouvons conclure que le rayonne- ment continu analysé s'interprète correctement $(10 \%)$ à partir de la théorie de W. Finkelnburg et Th. Peters [1].

En désignant par $n_{0}$ la densité des atomes neutres d'argon, l'équation de Saha [2] s'écrit :

$$
\log _{10} \frac{n_{\mathrm{e}}^{2}}{n_{0}}=-\frac{7,94 \times 10^{4}}{T_{\mathrm{s}}}+\frac{3}{2} \log _{10} T_{\mathrm{s}}+16,4 .
$$


En remplaçant la valeur de $n_{\mathrm{e}}^{2}$ dans l'expression (2) et en désignant par $N_{0}$ le nombre total d'atomes neutres contenus dans le plasma, nous obtenons :

$$
\begin{aligned}
\log _{10} \frac{P_{\mathrm{RV}}}{n_{0}} & =\log _{10} T_{\mathrm{s}}-\frac{7,94.10^{4}}{T_{\mathrm{S}}}+\text { Cte } \\
& =\log _{10} \frac{P_{\mathrm{R}}}{N_{0}} .
\end{aligned}
$$

Gette relation nous indique dans l'intervalle spectral envisagé que la puissance continue rayonnée par le plasma d'argon par atome n'est fonction que de sa température.

IV. Conclusion. - Dans un domaine où le débit d'argon est compatible avec le bon fonctionnement de la décharge $\left(Q>1 \mathrm{~m}^{3} / \mathrm{h}\right.$ environ), la puissance électrique $P_{\mathrm{E}}$, telle que nous l'avons définie, est reliée d'une manière biunivoque à la puissance à l'inducteur, donc à la puissance totale $P_{\mathrm{T}}$ de la décharge. Nous l'avons donc utilisée comme puissance de référence car sa mesure est facile et commode.

La puissance calorimétrique $P_{\mathrm{K}}$ est pratiquement indépendante du débit d'argon et pour des valeurs de $P_{\mathrm{K}}$ comprises entre 2 et $4 \mathrm{~kW}$ la courbe de variation en fonction de $P_{\mathrm{E}}$ est une droite. Pour les faibles valeurs, cette courbe a tendance à s'infléchir vers le bas. La mesure de $P_{\mathrm{K}}$ nous a permis de calculer la température moyenne calorimétrique des gaz de la décharge $T_{\mathrm{K}}$ dont la valeur très inférieure $(30 \%)$ à la température moyenne $T_{\mathrm{S}}$ déterminée par des méthodes spectrales met en valeur l'hétérogénéité de la décharge par rapport aux atomes d'argon. Remarquons, en outre, que $T_{\mathrm{K}}$ représente une valeur moyenne plus exploitable dans le cadre de réactions faisant intervenir l'ensemble des gaz de la décharge.

La puissance rayonnée diminue avec le débit d'argon et cette variation est pratiquement constante et égale à $11 \%$ pour une variation de $32 \%$ de ce dernier. Les différentes valeurs de la puissance rayonnée en fonction de la température montrent que, dans l'intervalle spectral $4500 \AA$ à $6500 \AA$, le rayonnement continu du plasma vérifie d'une façon satisfaisante $(10 \%)$ la théorie de W. Finkelnburg et Th. Peters [1]. Dans ces conditions, la puissance continue rayonnée par atome d'argon n'est'fonction que de la température du plasma.

Enfin, la température du plasma mesurée par des méthodes spectrales est, d'une part, pratiquement indépendante du débit d'argon et, d'autre part, sa variation est faible $(10 \%)$ par rapport à celle de la puissance électrique $(110 \%)$.

Manuscrit reçu le 11 mai 1967.

\section{BIBLIOGRAPHIE}

[1] Finkeinburg (W.) et Peters (Th.), Kontinuierliche Spektren, Handbuch der Physik, Springer-Verlag, Berlin, 1957, 28, 79.

[2] DELCROIX (J.-L.), Physique des Plasmas, $1^{\text {re édition, }}$ Dunod, Paris, 1963, 1, 23.

[3] Morris (A. D.), Conference on Extremely High Temperature, éditeurs H. Fischer et L. C. Mansur, New York, 1958, p. 45.

[4] Grawe (G. E.), Johnson (R. C.) et KRAUSE (L. N.), NASA TND-870, 1961.

[5] Fingerson (L. M.) et Blackshear (P. L.), U.S. Dep. Comm. AD, 258344, 1961.

[6] Grey (J.), Jacobs (P. F.) et Sherman (M. P.), Rev. Sci. Instr., 1962, 33, 738.

[7] Grey (J.), Rev. Sci. Instr., 1963, 34, 857.

[8] Haas (F. C.) et Vassalio (F. A.), Chem. Eng. Progr. Symp. Ser., 1963, 59, 165.

[9] Zsombor-Murray (P. J.), Mech. Eng. Res. Report 63-6, McGill University, 1963.

[10] TANkIn (R. S.) et BERRy (J. M.), Phys. Fluids, 1964, 7, 1620.

[11] Kasahara (E.), Hasur (O. A.), EMORI (Y.) et IrYOSHI (A.), Bulletin of J.S.M.E., 1964, 7, 59.

[12] Smith (D. L.) et ChurchiLI, (S. W.), Dept. of Chemical and Metallurgical Engineering Report 05607.1-T, University of Michigan, 1965.

[13] Cheng (D. Y.) et Blackshear (P. L. Jr), A.I.A.A., 2nd Annual Meeting paper 65-359, San Francisco, 1965 .
[14] KubaneK (G. R.), Thèse, Heat Transfer to Spheres and Cylinders in a Confined Plasma Jet, McGill University, 1966.

[15] BABAT (G.), Westnik Elektropromyshlennosty, 1942, 2 et 3.

BABAT (G.), Technika Molodeshi, 1942, 8.

BABAT (G. I.), J. Inst. Elec. Eng., 1947, 94, 27.

[16] REED (T. B.), J. Appl. Phys., 1961, 32, 821 et 2534.

REED (T. B.), Inter. Sci. Technol., 1962, 6, 42.

REED (T. B.), Proceedings of the National Electronics Conference, McCormick Place, Chicago, Illinois, 1963, 19, 654 .

[17] LAROCHE (J.), Thèse, Recherches physiques et chimiques sur l'excitation des gaz dans la torche électronique. Étude de la formation du bioxyde d'azote, Paris, 1961.

[18] Marynowsky (G. W.) et Monrof (A. G.), International Symposium on High Temperature Technology, Asilomar, California, 1963.

[19] Abravanes (G.), Thèse, Contribution à l'étude de la décharge haute fréquence. Application au dosage des métalloïdes dans les métaux, Toulouse, 1966.

[20] Dresvin (S. V.), Donskor (A. V.) et Gol'DFarb (V. M.), J. Technical Physics, 1965, 35, 1646.

[21] Dimchitz (B. M.) et Koretski (J. P.), J. Chemical Physics, 1965, 34, 1677.

[22] Besombes-VaIlhé (J.), J. Chim. Physique, 1967, 2, 370 . 
[23] DAUVERgne (J.-P.), Thèse, Étude de la sublimation et de la condensation de vapeurs d'oxydes réfractaires dans un four à plasma, Nancy, 1965.

[24] Yovanovitch (J.), L'usine nouvelle, 1965, 12.

[25] Adams (W. H. Mc), Transmission de la chaleur,

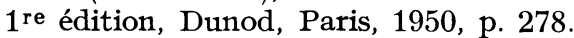

[26] KERN (D. Q.), Process Heat Transfer, $1^{\text {re }}$ édition, McGraw Hill book Company, New York, 1950, p. 73.

[27] Sy (A.-A.), Revue des hautes températures et réfractaives, 1964, 2, 1965.

[28] Lavalí́E (H.-Cl.), Thèse, Fonctionnement d'un jet de plasma à l'argon, Laval, 1965.

[29] Gol'Dfarb (V. M.) et Dresvin (S. V.), Teplofiz Vysokikh Temperatur, Akad. Nauk SSSR, 1965, 3, 333.

[30] Gol'DFarb (V. M.), Optika i Spektroskopiya, 1965, 19, 284.

[31] Borovick (E. S.), MiTin (R. V.) et KNIAZEFF (U. R.), Zh. Tekn. Fy., 1961, 31, 1329.

[32] Mitin (R. V.) et Pryadjin (K. K.), Zh. Tekn. Fy., 1965, 35, 1205.

[33] Triche (H.), C. R. Acad. Sci., 1957, 245, 1718. Triche (H.), C. R. Acad. Sci., 1959, 248, 2743. Triche (H.), C. R. Acad. Sci., 1963, 256, 907.
[34] Cabannes (F.), Chapelie (J.) et Sy (A.), C. $R$. Acad. Sci., Paris, 1964, 259, 1714.

[35] Cabannes (F.), Chapelie (J.) et Sy (A.), C. $R$. Acad. Sci., Paris, 1965, 260, 3320.

[36] VAN WeRMEIREN (R.), Spectroscopie des plasmas, Université de Bruxelles, Institut de Physique service électricité, 1964, 10.

[37] Busz (G.) et Finkeindurg (W.), Z. Physik, 1954, 139, 212.

[38] Gaun', Proc. Roy. Soc., A., 1930, 126, 654.

[39] Denisse (J.-F.), J. Physique Rad., 1950, 11, 164.

[40] Biberman (L. M.) et Norman (G. E.), Optika $i$ Spektroskopiya, 1960, 8, 433.

[41] Kramers (H. A.), Phil. Mag., 1923, 46, 386.

[42] OSTer (L.), Rev. Phys., 1961, 33, 527.

[43] Sy (A.-A.), Revue des hautes températures et réfractaires, 1964, 1, 295.

[44] DRELIISHAK (K. S.), KNOPP (C. F.) et CAMBEL (A. B.), Phys. Fluids, 1963, 6, 1280.

[45] Reboux (J.), Ingénieurs et techniciens, 1964, 177, 6.

[46] M.E.C.I., Catalogue F4, Mesures électriques des températures, Éditions Paul-Martial, Paris, 1963.

[47] Sherman (M. P.), Jacobs (P. F.) et Grey (J.), Aerospaces Research Laboratories, Report Arl 64132, Office of Aerospace Research U.S.A.F., 1964.

[48] MarlotTe (G. L.), HARder (R. L.) et PRIChard (R. W.), U.S. Dep. Comm. AD-6105, 45, 1964. 